\title{
Interactive comment on "Lagrangian simulations of the transport of young air masses to the top of the Asian monsoon anticyclone and into the tropical pipe” by Bärbel Vogel et al.
}

\section{Anonymous Referee \#3}

Received and published: 25 September 2018

The manuscript "Lagrangian simulations of the transport of young air masses to the top of the Asian monsoon anticyclone and into the tropical pipe" investigates transport pathways in the monsoon region from the boundary layer into the stratosphere. The authors use both Lagrangian backward trajectory calculations and three-dimensional simulations including irreversible mixing with the Lagrangian transport model CLaMS. Artificial tracers of air mass origin are compared to measurements of chlorodifluoromethane (HCFC-22; CHCIF2) by the Michelson Interferometer for Passive Atmospheric Sounding (MIPAS). The methods are similar to those in Vogel et al. (2016) but in addition to horizontal transport they address vertical transport pathways out of 
sphere. The chosen period is a normal monsoon season in terms of medium rainfall over India in summer 2008. The paper presents an interesting description of the "spiralling staircase". Consistency with MIPAS data supports the model results. The paper is well written and I support publication after a few comments have been addressed. The heavy focus on a particular day is didactical, but the analysis of different meteorological situations would provide stronger evidence. In certain passages the phrasing could be improved in order to more clearly distinguish what is the particular contribution of this study to the "longstanding debate about the transport mechanisms at the top of the Asian monsoon anticyclone and beyond into the stratosphere". The debate is mentioned again in the discussion but the different arguments about "the exact transport mechanism" could be more clearly stated. Otherwise it is difficult to distinguish if some very general assertions have been stated before in the literature or are novel to this work. The figures could be improved and the description thereof clarified in the text.

Minor comments:

p1 119 "However, this upward transport": Make "this" more clear (the upward spiralling range).

p2 I20 Briefly mention the sides in the debate and state where the authors stand.

p5 I3 Zzeta < 120K? p5 I 17 "is quantified." What will be the quantitative measure?

p6 I8 Dee et al. (2011) already cited in the first reference to ERAI in p4 I11

p7 I20 make more explicit the dates of the monsoon and the pulse releases to clarify the 6 month age.

122 "have strong variability from day to day" please rephrase

p8 11 mention the 360 theta level before in the text to streamline the reading.

Printer-friendly version

p8 110 "simulated horizontal gradients": is there an objective metric or visual inspection? How is the top of the asian monsoon precisely defined? 
p8 117 Fig. 2 (3rd row), to be consistent with previous paragraph.

p10 I15 When are the trajectories started? How many? What release pattern? It would help for understanding what was done to state this clearly in the text. 40 days could be analysed statistically, bur for individual trajectories is a little bit too long.

p10 I20 describe the panels of fig 5 in the text.

p10 last paragraph. At this point the reader would feel satisfied with a statistical analysis of a larger number of days to support the case study results. Maybe some additional results such as those later presented in A1 could be mentioned.

p11 I4 what do you mean with "single selected trajectories"?

p11 I32 "In the previous sections, we could show that the Asian monsoon is an effective circulation pattern in the UTLS that transports very young air masses ( $<6$ months) from the surface into the lower stratosphere up to $\approx 460 \mathrm{~K}$." This may have been mentioned before in the literature. You could rephrase this as "we could show how the effective circulation pattern in the UTLS can be seen with CLaMS and MIPAS data, for example. Also it seems that all your conclusions will be drawn from a single day case study. Additional statistical evidence from more modelling cases could help.

124 is it a CLaMS simulation?

Fig 8. The figure is difficult to read. The label at the color bar is very small and takes time to find. You could replace the title of the subplots "08081812 at $90 \mathrm{E}$ " that is the same for all and put is in the cation. "horizontal winds (black lines)", do you mean horizontal wind absolute value isolines? "corresponding levels of pressure "corresponding to what? "Pressure levels as white lines" would be better. Are the values really zero in W07 (lower left panel) or is the color scale?

Printer-friendly version

"demonstrating" may sound a bit strong for this context. "Suggesting" or "indicating" could fit better. 
p12 I14 If still describing Fig. 9 better to keep the same paragraph.

Figure 10: again, wind speed contours, "pressure levels" instead of "corresponding levels of pressure"

p13 111: attract the attention of the reader to the vertical dashed line immediately here.

Interactive

Fig 13 what is the dashed line?

comment

p15 I12 please refer to the published version.

p16 117 "Thus, air masses in the upward spiral range are uplifted by diabatic heating across the (lapse rate) tropopause, which does not act as a transport barrier against this diabatic vertical transport process.: This assertion is likely to be "consistent with previous studies".

p16 I 22 occurs where?

The last paragraph is a nice summary of the mechanism but it undoubtedly draws from the conclusions of many previous studies. This should somehow be acknowledged.

Interactive comment on Atmos. Chem. Phys. Discuss., https://doi.org/10.5194/acp-2018-724, 2018. 